thickness were significantly larger in 116 patients with RCM than in normal subjects $(p<0.05)$. However, there were no statistical differences between the two groups in left ventricular diastolic diameter (LVDD). Visual observation showed that mild mitral regurgitation $(43 \%)$, moderate mitral regurgitation (21\%), mild tricuspid regurgitation (28\%) and severe tricuspid regurgitation (40\%) were noted, respectively. 35 RCM with DE was further divided into diffuse and segmental enhancement. RCM with diffuse delayed enhancement was 15 cases, of which 12 cases showed powdery enhancement, and three showed petaline enhancement. Three cases with powdery enhancement were histologically proven as myocardial amyloidosis. RCM with segmental enhancement was 20 cases. Ventricular septum was the most vulnerable segment. Six cases presented subendocardial enhancement that corresponded to apical obliteration, of which one case was confirmed as hypereosinophilia with use of marrow examination. The other 14 cases didn't present any regular enhancement. 81 RCM without DE, of which histologically proven non-specific findings were in two cases, had marked bi-atrial dilation, near-normal ventricular chambers and near-normal ventricular thickness.

Conclusions MRI is an excellent imaging modality to identify restrictive cardiomyopathy. Primary RCM presents marked bi-atrial dilation with nonhypertrophied and nondilated ventricles. Diffuse left ventricular thickening associated with powdery enhancement indicates myocardial amyloidosis. Apical obliteration associated with subendocardial enhancement corresponds to endomyocardial fibrosis.

\section{e0234 THE EFFECT OF LUTEOLIN ON $\mathbf{H}_{2} \mathbf{O}_{2}$-INDUCED VASCULAR SMOOTH MUSCLE CELL PROLIFERATION AND MIGRATION}

\section{doi:10.1136/hrt.2010.208967.234}

Chen Dan, Li Dongye, Lang Yasong, Zhu Manyi, Zhu Hong, Oian Wenhao, Luo Yuanyuan. Affiliated Hospital of Xuzhou Medical College, Xuzhou, Jiangsu, China

VSMCs Migration and proliferation are the critical pathological processes of various cardiovascular disease, such as atherosclerosis. Luteolin is a kind of flavonoids naturally occurring in many vegetables, fruits and medical plant. In this study, we investigated the effect of luteolin on the proliferation and migration of rat vascular smooth muscle cells (VSMCs) which were stimulated by $\mathrm{H}_{2} \mathrm{O}_{2}$, and a primary discussion was given to its mechanism of action. The present study demonstrated that Luteolin $(12.5$ to $50 \mu \mathrm{M})$ showed a particularly concentration-dependent inhibition effect on $\mathrm{H}_{2} \mathrm{O}_{2}$-elicited VSMCs proliferation and migration by MTT and Transwell assay respectively. In further research, we originally explored the function of Luteolin in blocking $\mathrm{H}_{2} \mathrm{O}_{2}$-triggered Src and Akt signalling pathways. The activation of Src, PDK1, Akt (308), Akt (473) of Luteolin group was significant lower than that of $\mathrm{H}_{2} \mathrm{O}_{2}$ group. These findings strongly suggested that Luteolin suppressed $\mathrm{H}_{2} \mathrm{O}_{2}$-directed migration and proliferation in VSMCs by inactivating Src and Akt pathways which participate in VSMCs migration and proliferation.

\section{e0235 STUDY ON THE PRIMARY AND SECONDARY PROTEIN STRUCTURE OF ISCHAEMIA MODIFIED ALBUMIN}

doi:10.1136/hrt.2010.208967.235

Shen Xiaoli, Han Lili, Xing Chao, Lin Lin, Lin Saimei, Pan Leng, Liu Xiaoqing, Pu Xiaodong, Deng Yulian. Affiliated Fujian Provincial Hospital, Fujian Medical University/Fujian Provincial Key Laboratory of Cardiovascular Disease

Objective Ischaemia-modified albumin (IMA) has been demonstrated to be a biomarker of ischaemia associated with myocardial and skeletal muscle ischaemia, pulmonary embolism and stroke. However there is limited information on the formation mechanism of IMA. The aim of the study was to investigate the primary and secondary protein structure of IMA.

Methods This study included 29 acute coronary patients (IMA level $>0.8$ absorbance units) and 22 healthy controls (IMA level $<0.5$ absorbance units). Serum IMA was purified by salting out and ion exchange chromatography. We also chose 21 human albumin standard. The structures of purified protein and albumin standard were analysed by mass spectrometry, $\mathrm{N}$-terminal sequencings and circular dichroism (CD) spectra measurement.

Results Protein sequences showed that the first $10 \mathrm{~N}$-terminal amino acid residues of IMA were identical with those of albumin in healthy persons. The result of $\mathrm{CD}$ spectra measurement revealed that the average percentages of $\alpha$-helixes and random coil decreased and the average percentages of $\beta$-turn and $\beta$ pleated sheets increased a bit in ACS patients, but there is no significant difference between groups.

Conclusion Compared with the normal human albumin, no changes take place in the $\mathrm{N}$-terminal protein sequence of IMA, and the secondary structure of IMA was also not significantly changed. Increasing percentage of $\beta$-turn and $\beta$ pleated sheets in IMA may correlate with its formation mechanism.

Acknowledgements This work was supported by grants from Science and Technology Planning Program of Fujian Province (2005D048) and Medicine Innovation Program of Health Department of Fujian Province (2003-CX-2).

\section{e0236 A REPERFUSION MODEL IN AMI RABBITS}

doi:10.1136/hrt.2010.208967.236

${ }^{1}$ Zhang Jun, ${ }^{2}$ Oi Xiao-yong, ${ }^{1}$ Wan Yan-fang, ${ }^{1}$ Yuan Chen, ${ }^{1}$ Ma Zhan-feng, ${ }^{1}$ Wang Ning, ${ }^{2}$ Dang Yi, ${ }^{1}$ Yuan Bo-min. ${ }^{1}$ Department of Cardiology, Cangzhou Central Hospital, Affiliated Hospital of Hebei Medical University, Cangzhou, China; ${ }^{2}$ Department of Cardiology, Hebei General Hospital, China

Objective To Explore the feasibility of establishing the reperfusion model on AMI rabbit by the method of obstructing and releasing the Left Anterior Ventricular Branch (LAVB) of left circumflex coronary artery (LCX).

Methods A total of 24 healthy Japanese albino rabbits of both sex were used in this study. Rabbits were randomly divided into 3 groups: ischaemia-reperfusion group (IR group, 8), ischaemianoperfusion group (AMI group, 8), sham group (sham, 8). After preconditioning the myocardium twice by obstructing the blood flow for $5 \mathrm{~min}$, we obstructed the flow of LAVB in IR group for $60 \mathrm{~min}$, and then released it to be reperfusion. In AMI group we obstructed the flow permanently by ligating LAVB. And in sham group we only threaded but did not obstruct the flow. Then we killed them 3 days later. Venous blood was gathered. The levels of cTNI, CK and CK-MB were assayed at pre-reperfusion (baseline) and post-reperfusion period (4-h, 8-h, 12-h, 24-h, 48-h and 72-h after being reperfusion). The summation changes of ST segment elevation were observed in leads $\amalg, \amalg$, avF by ECG. Histopathology of myocardia, Evan's Blue and TTC dyeing were taken into notice. STATA8.0 software pack was used for data analysis.

Results The results come out that the ST segment all elevated in each group after LAVB was obstructed. In IR group the ST segment lowered more than $50 \%$ within 120 min after releasing the artery. This phenomenon had not appeared in other two groups. Compared to baseline, the cTNI, CK and CK-MB were all raised in IR group, and the peak value antedisplaced to $8 \mathrm{~h}, 12 \mathrm{~h}$ and $10 \mathrm{~h}$. These three factors were all raised but no antedisplacement of the enzyme peak in AMI group. In sham group the raising of the three factors was slight, and no antedisplacement of the enzyme peak either. The experiment of rabbits in IR and AMI groups were consistent with the AMI diagnostic criteria in AMI Guideline of diagnosis and therapy established by Cardiac Disease branch of Chinese Medical 
Association in 2004. The histopathology examination and TTC staining agree with the AMI diagnosis too. The experiment of rabbits in IR group was in accordance with the recanalisation criteria of IRCA recommended by the Chinese J Cardiol editorial committee in 1991. The injection of Evan's Blue was in coincidence with the recanalisation of IRCA too.

Conclusions AMI-reperfusion rabbit models can be successfully established by the application of this method. It has proved to be very effective.

\section{E0237 EXTRACELLULAR MATRIX ISOLATED FROM MYOCARDIAL TISSUE SUPPORTS THE CARDIOMYOCYTE DIFFERENTIATION OF EMBRYONIC STEM CELLS}

doi:10.1136/hrt.2010.208967.237

Ou Dongbo, Zheng Diangsun, He Yong, Wang Zikuan. Department of Cardiology, Tangdu Hospital Fourth Military Medical University, Xi'an, China

Objective Embryonic stem cells (ESCs) represent an extremely promising cell source for tissue-engineered myocardial tissue. But the role of extracellular matrix in control and guidance of ESCs differentiation and commitment into complex and viable threedimensional (3D) myocardial tissue is still poorly understood. The purpose of this study was to identify and functionally characterise survive and differentiation of ESCs in 3D extracellular matrices isolated from myocardial tissues.

Methods Undifferentiated ESCs were cultured as previously described. Native myocardial tissues isolated from hearts of adult rabbit were treated with trypsin-EDTA and $\mathrm{NaOH}$ to remove cell components to gain acellular matrices. The acellularization of myocardial tissues was documented by haematoxylin-eosin staining (HE staining). Then ESCs were seeded onto the acellular matrices. Cell density and distribution were assessed by HE staining, cardiac transcription factors such as GATA4, NKX2.5, and atrial natriuretic factor (ANF) were evaluated by RT-PCR, and cardiac markers such as $\boldsymbol{\alpha}$-actinin, troponin I, and connexin 43 were evaluated by immunocytochemistry. Ultrastructural analysis was examined under a transmission electron microscope.

Results The acellular matrices were incompletely covered by cells at 1 week, and the interstitiums of matrices were densely repopulated by cells at 2 weeks post seeding, indicating the rapid growth and expansion of these cells in the extracellular matrices and the formation of live constructs. Cardiac transcription factors GATA4 and NKX2.5 began to express on day10, but the expression of ANF was detected on day 12 . The constructs were positively immunostained with $\alpha$-actinin, troponin I, and connexin 43 at 2 weeks post seeding. Ultrastructural analysis indicates that cells in constructs exhibited the morphological features including sarcomeres, abundant glycogen and mitochondria and nascent junctional complexes. Conclusions These results indicate that extracellular matrices have an important role to promote the differentiation of embryonic stem cells into cardiomyocytes. Such cell differentiation with extracellular matrices may be useful in forming tissue-engineered myocardial tissue to repair specific damaged hearts.

\section{e0238 EFFECTS OF COMBINED ATORVASTATIN/COENZYME 010 ON MYOCARDIAL FIBROSIS AND MALONDIALDEHYDE (MDA) AND SUPEROXIDE DISMUTASE (SOD) IN RAT OF HEART FAILURE}

doi:10.1136/hrt.2010.208967.238

${ }^{1}$ Zhao Dongming, ${ }^{2}$ Yang Ping. ${ }^{1}$ Affiliated Hospital of Beihua University; ${ }^{2}$ China-japan Union Hospital of Jilin University

Objective To observe the effects of Combined Atorvastatin/Coenzyme Q10 on myocardial fibrosis and MDA and SOD in Rat of heart failure, to explore the protective effect of Combined Atorvastatin/ Coenzyme Q10 on the heart of the rat.

Methods Female Wister rats were subjected to the ligation of the anterior descending branch of the left coronary artery or sham operation during halothane anaesthesia. The rat heart failure models were established after 6 weeks. These rats were divided into four groups ( $n=6$ each group): Sham operated group; model group; model group-atorvastatin, Rats in this treatment group were administrated by gastric perfusion of atorvastatin $(10 \mathrm{mg} / \mathrm{kg} /$ day); model group-atorvastatin-Coenzyme Q10. Rats in this treatment group were administrated by gastric perfusion of atorvastatin $(10 \mathrm{mg} / \mathrm{kg} /$ day) and Coenzyme Q10 (30 mg/kg/day). All treatment regimens were initiated 6 weeks after surgery and continued for 5 weeks. After Masson stain, myocardial fibrosis of these rats were observed by light microscope. The activity of serum SOD and the content of serum MDA were detected by biochemical methods.

Results Compared with that in model group, myocardial fibrosis level and MDA content was decreased in model group-atorvastatin $(p<0.01)$. At the same time, the activity of SOD was increased $(p<0.01)$. Compared with that in model group-atorvastatin, myocardial fibrosis level and MDA content was descreased in model group-atorvastatin-Coenzyme $\mathrm{Q} 10(\mathrm{p}<0.05)$. At the same time, the activity of SOD was increased $(\mathrm{p}<0.05)$.

Conclusion Compared with atorvastatin, Combined atorvastatin/ coenzyme Q10 could protect the myocardium further, and MAD and SOD maybe were involved in this process.

\section{e0239 N-TERMINAL PRO-BRAIN NATRIURETIC PEPTIDE (NT- PROBNP): A POTENTIAL DIAGNOSTIC BIOMARKER FOR PREDICTING CARDIAC DYSFUNCTION IN PATIENTS WITH LIVER DISEASES}

doi:10.1136/hrt.2010.208967.239

${ }^{1}$ Wang Ling, ${ }^{1}$ Geng Jiabao, ${ }^{1}$ Chang Yibin, 'Li Tong, ${ }^{2}$ Matsumori Akira, 'Zhuang Hui. ${ }^{1}$ Peking University Health Science Center; ${ }^{2}$ Department of Cardiovascular Medicine Kyoto University Japan

Background NT-proBNP has emerged as a powerful diagnostic and prognostic biomarker in heart disease. Studies showed that NTproBNP is a sensitive biomarker for identifying patients with heart failure caused by hepatitis $\mathrm{C}$ virus (HCV) related myocarditis. The aim of this study was to investigate the relationship between the elevated concentration of NT-proBNP and liver diseases.

Methods 223 serum samples from blood donors were collected as a control group, and 644 samples were obtained from patients infected by hepatitis viruses including 493 HBV (364 chronic hepatitis B, 86 hepatocellular carcinoma and 43 liver cirrhosis) and $151 \mathrm{HCV}$. All samples were assayed with an Elecsys immunoassay analyser for NT-proBNP concentration.

Results The mean concentration of NT-proBNP in the control group was $21.77 \pm 15.56 \mathrm{pg} / \mathrm{ml}$ which showed no significant variation with age or gender. Both the mean value and the rate of abnormality of NT-proBNP were significantly higher for the HBV and HCV infected groups as compared to the control group. The mean NT-proBNP value $(380.24 \mathrm{pg} / \mathrm{ml})$ and abnormality rate $(38.41 \%)$ in the HCV group were higher than that of HBV group. For samples from patients with HBV related hepatic disease/pathology, the mean NTproBNP value $(517.19 \mathrm{pg} / \mathrm{ml})$ and abnormality rate $(58.14 \%)$ were the highest in the liver cirrhosis group.

Conclusions The elevation of NT-proBNP in patients with progressed liver disease in this study suggested the presence of cardiac dysfunction. 